\title{
Problems of modelling Proportional-Integral- Derivative controller in automated control systems
}

\author{
Anna Doroshenko* \\ Moscow State University of Civil Engineering, Yaroslavskoye shosse, 26, Moscow, Russia, 129337
}

\begin{abstract}
The actual task of modelling automatic control systems is to use control algorithms that include some function that limits the output signal. Proportional-integral-derivative (PID) controllers are used to control dynamic processes with variable at wide limits parameters and uncontrolled violations in automated process control systems. Despite the wide distribution, this type of controllers is not easy to configure. In article the problems of modelling proportional-integral-derivative controller are discussed. The methods of combating the effect of integral saturation are studied. The results of using algorithms of combating the effect of integral saturation are represented.
\end{abstract}

\section{Introduction}

The actual task of modelling automatic control systems is to use control algorithms that include some function that limits the output signal.

Proportional-integral-derivative (PID) controllers are used to control dynamic processes with variable at wide limits parameters and uncontrolled violations in automated process control systems.

The PID controller was invented in 1910 [1]. In 1942, Ziegler and Nichols developed a method for its tuning, and after the advent of microprocessors in the 1980s the development of PID regulators was taking place at an increasing rate [2].

The PID controller is one of the most common type of controllers. About $90-95 \%$ of the controllers currently in operation use the PID algorithm [3]. The reason for such high popularity is the simplicity of construction and industrial use, the clarity of functioning, the suitability for solving most practical problems and low cost.

The PID controller has received wide application in various in purpose and design systems of automatic control of the working parameters of equipment, which in turn makes it easy and easy to provide signal generation [4]. The ultimate goal is to obtain high data accuracy, as well as the quality level of the transient process itself [5-8].

The PID controller uses the proportional-integral-differential regulation law. The PID controller usually has additional service properties of automatic tuning, signalling, self-

* Corresponding author: pochta.avd@gmail.com 
diagnostics, programming, unstressed switching modes, remote control, the ability to work in an industrial network, etc [9].

Some of the problems arise because of the complexity of operation. In many PID controllers, the differential component is disabled only because it is difficult to properly configure. Users neglect the calibration procedure, insufficiently thorough knowledge of the dynamics of the controlled process does not allow choosing the correct parameters of the regulator. As a result, $30 \%$ of the regulators used in the industry are set up incorrectly. Therefore, the main efforts of researchers are currently focused on the search for reliable methods for automatic adjustment of regulators, both integrated in the PID controller, and functioning on a separate computer.

After the emergence of cheap microprocessors and analogue-to-digital converters PID controllers use automatic parameter adjustment, adaptive algorithms, fuzzy logic methods, genetic algorithms are used. The structure of the controllers became more and more complicated [1-39].

\section{PID controller parameters}

Despite the wide distribution, this type of controllers is not easy to configure, since it is necessary to search by three parameters: proportional (kp), integral (ki) and differential (kd) components [10-12]. The transfer function of these regulators is usually represented as follows:

$$
W(s)=k_{p}\left(1+\frac{1}{T_{i} s}+T_{d} s\right)=k_{p}+k_{i} \frac{1}{s}+k_{d} s
$$

At present, there is still no single method for calculating the parameters of the controller for the control loop. The most famous methods are the empirical Ziegler-Nichols method (the method of undamped oscillations), the damping oscillation method, the Kuhn method, the Schädel method, etc. Each of the methods has advantages and disadvantages and is applied under certain requirements to the regulatory system. The choice of method directly depends on the control object.

The actual task for modeling automatic control systems is to use control algorithms that include some function that limits the output signal.

The equation of a nonlinear element of saturation type is represented by the formula:

$$
\bar{u}=\left\{\begin{array}{l}
u_{\min }, u<u_{\min } \\
u, u_{\min } \leq u \leq u_{\max } \\
u_{\max }, u>u_{\max }
\end{array}\right.
$$

Integral saturation is the effect that is observed when the PI or PID controller has to compensate for a long time an error outside the range of the controlled variable, since the controller output is limited, it is difficult to reduce the error to zero.

If the control error persists for a long time, the value of the integral component of the controller becomes very large. This, in particular, occurs if the control signal is limited enough that the calculated output of the controller is different from the actual output of the actuator. Since the integral part becomes zero only a short time after the error value changed sign. Integral saturation can lead to a large overshoot. 
In order to avoid this effect in the modeling of PID regulators in automated control systems in software complexes, algorithms for protection against integral saturation are laid down.

\section{PID controllers modelling}

In order to avoid this effect in the modeling of PID regulators in automated control systems in software complexes, algorithms for protection against integral saturation are laid down.

For example there are two built-in protection algorithms in Matlab Simulink: BackCalculation (Figure 1) and Clamping (Figure 2).

Simulink is an interactive tool for modeling, simulating and analyzing dynamic systems. It provides the ability to build graphical block diagrams, simulate dynamic systems, investigate the operability of systems and improve designs. Simulink is fully integrated with MATLAB, providing immediate access to a wide range of analysis and design tools. These advantages make Simulink one of the most popular tool for designing control systems.

In the additional settings of the controller, you can set limits on the input signal. And when using this setting, you can configure protection against the integral saturation effect. This effect occurs when the actuator has a saturation limitation on the input. Because of this limitation, it is not possible to reach the set point, which results in a non-zero error value at the regulator input. During operation, the integrator continues to accumulate this error, the signal at its output increases, but does not participate in the regulation process (does not affect the object). As a result, there is a delay in the transient process. In order to avoid this effect, you must set the limit "Limit output" and choose one of the two methods of protection "Anti-windup method".

Algorithm "back-calculation". In this algorithm, the integral saturation effect occurs with the help of additional feedback, for the transmission of signals, the difference between the received control signal and taking into account the saturation at the integrator input is taken into account. Figure 1 shows the scheme of the "back-calculation" algorithm.

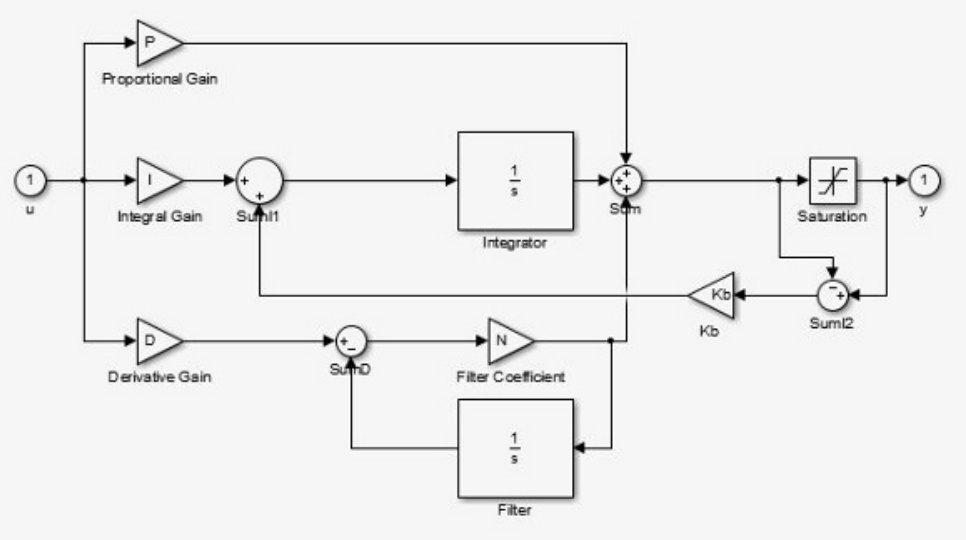

Fig. 1. Back-Calculation algorithm.

The back-calculation anti-windup method uses a feedback loop to discharge the PID Controller's internal integrator when the controller hits specified saturation limits and enters nonlinear operation.

Another commonly used anti-windup strategy is based on conditional integration. 


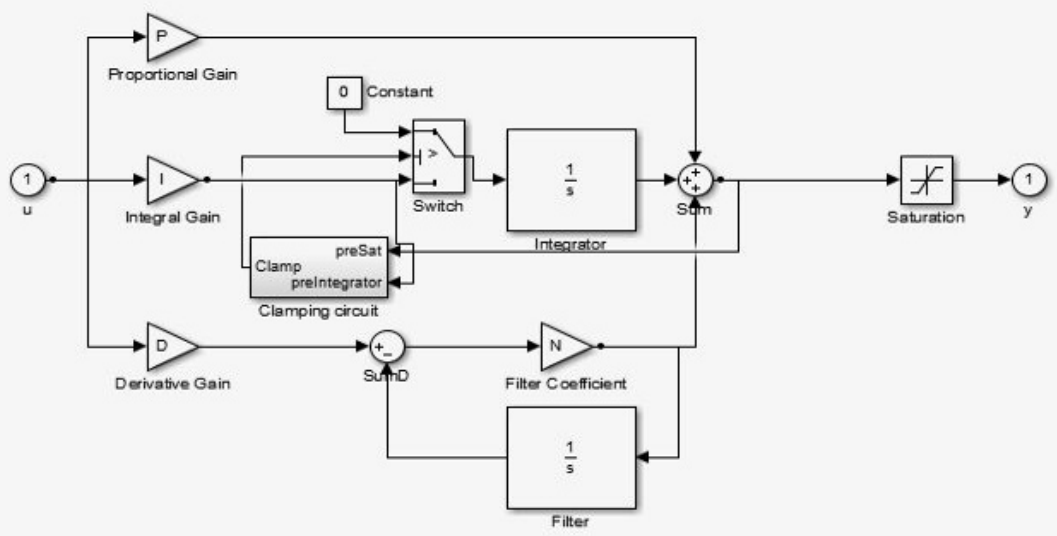

Fig. 2. Clamping algorithm.

When the algorithm changes, the structure of the PID controller changes. When the control action on the object reaches saturation, we programmatically disconnect the integrator from the control. The advantage of this method is that the nature of the transient process is much better, that is, it terminates faster and the regulator output does not exceed saturation.

Clamping will always work. It detects when there is an integrator overflow and sets the integral path of the PID controller to zero to avoid. Clamping is a commonly used antiwindup method, especially in the case of digital control systems. In serious applications, however, there is also forward clamping involved. This mechanism must be implemented manually.

Back Calculation highly depends on the back calculation coefficient $\mathrm{Kb}$. If the user doesn't know how to actually calculate the parameter Kb don't use back-calculation. This method calculates the difference between the actual controller output and the saturated output and subtracts it from the I-Gain path, amplified by Kb. In most of cases the default value $\mathrm{Kb}=1$ will lead to worse results than clamping, it is even possible that it has no effect at all. $\mathrm{Kb}$ should be calculated based on the sampling time or in case a D-Gain is involved, based on D- and I-Gain. Appropriate literature should be consulted to calculate the coefficient. Back calculation with a properly set coefficient enables better dynamics than clamping

Consider the results of using the back-calculation algorithm in the example with PID controller and without it (Figure 3). The results obtained demonstrate high efficiency of back-calculation algorithm use.
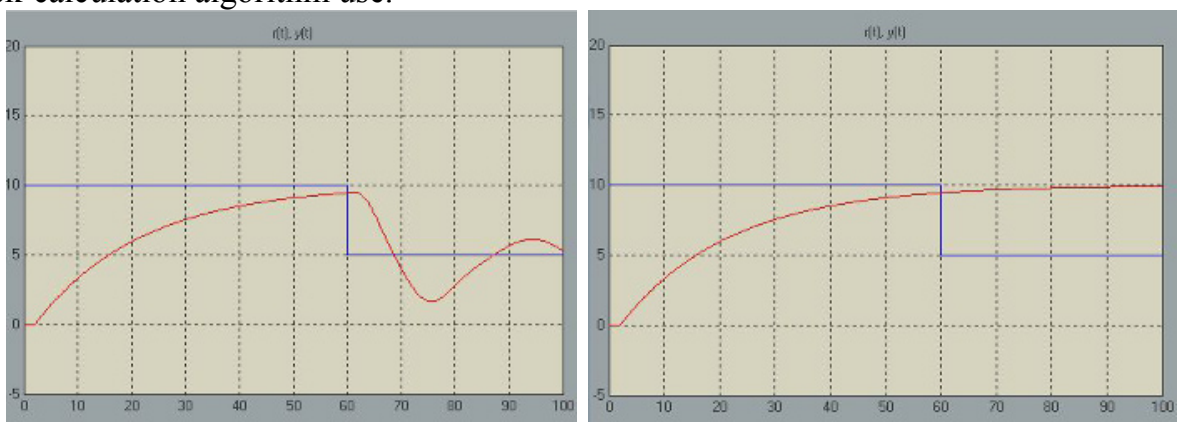

Fig. 3. The results of using the back-calculation algorithm - left figure, without using the back-calculation algorithm - right picture. 


\section{Discussion}

With a properly tuned PID controller, in most cases, it is possible to ensure that all system requirements are met. Because of their simplicity, they have received the widest distribution. According to statistics, more than $90 \%$ of all industrial regulators are PIDcontrollers.

Constantly growing market requirements to reduce the time of regulation, to the quality of the transient process, to the degree of weakening of the influence of external disturbances and noise, simplification of the tuning procedure and the need to control objects with a large transport delay initiated the appearance of many modifications of PID regulators.

Integral saturation is the result of nonlinearities in the system associated with limiting the output control. The negative effect of integral saturation causes a deterioration in the quality of regulation. Increase in regulation time and large overshoot. Under certain conditions, integral saturation can lead to self-oscillations, even if in the linear zone the control system ensures a high quality of regulation. Therefore, various ways have been developed to eliminate the effect of integral saturation, which do not allow the control signal to reach the boundary values and, nevertheless, ensure a good quality of transient processes. These methods are included in various simulation complexes of automatic control systems.

\section{Conclusions}

The use of integral saturation compensation algorithms has the following advantages: implementation of the method does not require powerful computing facilities and the quality of regulation is significantly improved.

When developing automatic control systems it is necessary to take into account the presence of some non-linearities and use algorithms for taking into account the limitations, depending on the available automation equipment, the complexity of the process and the technological requirements for it.

The use of integral saturation compensation methods in self-tuning systems of automatic control using adaptation and identification algorithms is promising.

\section{References}

1. M. Bertocco, S. Cappellazzo, A. Flammini, M. Parvis, IMTC/2002, 2, $1261-1264$ (2002)

2. J. Ziegler, N. Nichols, Trans. ASME, 64, 759-768 (1942)

3. K. Astrom, T. Hagglund, ISA, , 460p (2006)

4. S.A. Ajwad, , J. Iqbal, M.I.Ullah, A. Mehmood, Frontiers of Mechanical Engineering, 10 (2), 198-210 (2015)

5. N. Makisha, Pr. Eng., 165, 1092-1097 (2016)

6. A. Volkov, E. Batov, Pr. Eng., 111, 849-852 (2015)

7. A. Volkov, Advanced Materials Research, 838-841, 2969-2972 (2014)

8. L. Shirokov, P. Chelyshkov, E. Romanenko, MATEC Web of Conferences, 86, 04062 (2016)

9. A. Volkov, L. Sukneva, Applied Mechanics and Materials, 587-589, 338-341 (2014)

10. M. Miranda, K. Vamvoudakis, Proceedings of the American Control Conference, 5443-5448 (2016) 
11. Z. Yang, Y. Gao, D. Zhang, T. Huang, Key Engineering Materials, 238-239, 375-380 (2003)

12. A.J. Winsby, S.E. Burge, M.B. Widden, International Journal of Electrical Engineering Education, 41 (4), 292-306 (2004)

13. S. Skogestad, Journal of Process Control, 13 (4), 291-309 (2003)

14. Z.L. Gaing, IEEE Transactions on Energy Conversion, 19 (2), 384-391 (2004)

15. K.H. Ang, G. Chong, Y. Li, IEEE Transactions on Control Systems Technology, 13 (4), 559-576 (2005)

16. M.V. Kothare, P.J. Campo, M. Morari, C.N. Nett, Automatica, 30 (12), 1869-1883 (1994)

17. P. Ge, M. Jouaneh, IEEE Transactions on Control Systems Technology, 4 (3), 209-216 (1996)

18. Z. Gao, Proceedings of the American Control Conference, 6, 4989-4996 (2003)

19. R.W. Longman, International Journal of Control, 73 (10), 930-954 (2000)

20. C.C. Hang, K.J. Astrom, W.K. Ho, IEE Proceedings D: Control Theory and Applications, 138 (2), 111-118 (1991)

21. K.J. Åström, T. Hägglund, Journal of Process Control, 14 (6), 635-650 (2004)

22. A.I. Dounis, C. Caraiscos, Renewable and Sustainable Energy Reviews, 13 (6-7), 1246-1261 (2009).

23. Y.Q.Chen, I. Petráš, D. Xue, Proceedings of the American Control Conference, art. no. 5160719, 1397-1411 (2009)

24. H. Zhang, Y. Shi, A. Saadat Mehr, IEEE Transactions on Industrial Electronics, 58 (12), art. no. 5699920, 5396-5405 (2011)

25. M. Zamani, , M. Karimi-Ghartemani, N. Sadati, M. Parniani, Control Engineering Practice, 17 (12), 1380-1387 (2009)

26. F. Padula, A. Visioli, Journal of Process Control, 21 (1), 69-81 (2011)

27. Y. Li, Q. Xu, IEEE Transactions on Control Systems Technology, 18 (4), art. no. 5282525, 798-810 (2010)

28. W. Tan, IEEE Transactions on Power Systems, 25 (1), art. no. 5361327, 341-350 (2010)

29. Y. Luo, Y. Chen, Automatica, 45 (10), 2446-2450 (2009)

30. N. Makisha, E3sConf, 6, 01002 (2016)

31. A. Biswas, S. Das, A. Abraham, S. Dasgupta, Engineering Applications of Artificial Intelligence, 22 (2), 343-350 (2009)

32. L. Guo, J.Y. Hung, R.M. Nelms, IEEE Transactions on Industrial Electronics, 56 (6), 2237-2248 (2009)

33. P.E.I. Pounds, D.R. Bersak, A.M. Dollar, Proceedings - IEEE International Conference on Robotics and Automation, art. no. 5980314, 2491-2498 (2011)

34. H. Zhang, Y. Shi, A.S. Mehr, International Journal of Robust and Nonlinear Control, 22 (2), 183-204 (2012)

35. D.Q. Truong, K.K. Ahn, Mechatronics, 19 (2), 233-246 (2009)

36. I. Pan, S. Das, A. Gupta, ISA Transactions, 50 (1), 28-36 (2011)

37. Y. Luo, Y.Q. Chen, C.Y. Wang, Y.G. Pi, Journal of Process Control, 20 (7), 823-831 (2010)

38. R.J. Wai, J.D. Lee, K.L. Chuang, IEEE Transactions on Industrial Electronics, 58 (2), art. no. 5437232, 629-646 (2011)

39. M. Kano, M. Ogawa, Journal of Process Control, 20 (9), 969-982 (2010) 